\title{
Impacto del semáforo nutricional en los hábitos alimenticios de los estudiantes de la Universidad Politécnica Salesiana
}

\section{Impact of the nutritional semaphore on the eating habits of the students of the Salesian Polytechnic University}

\author{
Mariella Angelina Velasteguí Peñafiel \\ Universidad Politécnica Salesiana, Ecuador
}

Autor para correspondencia: mvelastegui@ups.edu.ec

Fecha de recepción: 30 de Agosto de 2016 - Fecha de aceptación: 25 de Octubre de 2016

Resumen: En Ecuador, el alto índice de defunción de personas con enfermedades no transmisibles como la diabetes, obesidad, hipertensión, entre otras, produjo que el Gobierno Nacional implementara el uso del etiquetado del semáforo nutricional en los productos procesados. Esta herramienta constituye una fuente de información para los consumidores sobre los niveles de grasas, azúcares y sales que contienen los productos. La iniciativa, tuvo como finalidad convertirse en una guía para modificar los hábitos alimenticios de los ecuatorianos. Dentro de este marco, conocer el impacto social que ha generado la implementación del semáforo nutricional en los hábitos alimenticios de los estudiantes de la Universidad Politécnica Salesiana sede Guayaquil, se convierte en la problemática planteada durante el siguiente trabajo investigativo. Las causas para la aplicación del semáforo nutricional son muy variadas y se determinarán a lo largo de la investigación. La importancia del presente artículo radica, en la necesidad de informar al público sobre el uso de este instrumento que ayudará a mejorar los hábitos alimenticios de los consumidores.

Palabras claves: semáforo nutricional; hábitos alimenticios; impacto; consumidores; implementación

\begin{abstract}
In Ecuador, the high index of death of people with non-transmissible illnesses such as: diabetes, obesity, hypertension, etc, made the National Government implement the use of the tagged nutritional traffic light in the processed goods. This tool constitutes the information source for consumers about the fat, sugar, and salt levels that certain products contain. The initiative had the goal of becoming the guide to modify the Ecuadorian's regular diet. Inside this environment, knowing the impact that the implementation of the nutritional traffic light has generated in the regular diet of the students of the Salesian Polytechnic University in Guayaquil, becomes the subject proposed during the following investigative work. The causes for the application of the nutritional traffic light varies and they will be determined along our investigation. The importance of this article resides in the necessity of informing the public about the use of this instrument which will help the users improve their regular diet.
\end{abstract}

Key words: nutritional traffic light; regular diet; impact; consumers; implementation 


\section{Introducción}

En el continente americano existe un elevado índice de personas que sufren enfermedades no transmisibles debido al consumo excesivo de productos altos en sales, grasas y azúcares. La preocupación dentro de este contexto ha llevado a la implementación de una herramienta que ayude a reducir este indicio.

En Ecuador, según estimaciones realizadas en el 2011 por el Instituto Nacional de Estadística y Censos (INEC), la diabetes mellitus tipo 2, la enfermedad hipertensiva y la enfermedad cerebrovascular fueron las principales causas de muerte. Esas enfermedades crónicas no transmisibles están asociadas casi en su totalidad con el sobrepeso y la obesidad, provocados por el consumo elevado de alimentos poco nutritivos, con alta densidad energética y alto contenido de grasa, azúcar y sal, así como por la reducción de la actividad física y el consumo de tabaco. Zurita, A. (19 de abril del 2015). Rojo, amarillo y verde: Tres colores que no lo dicen todo. El Universo.

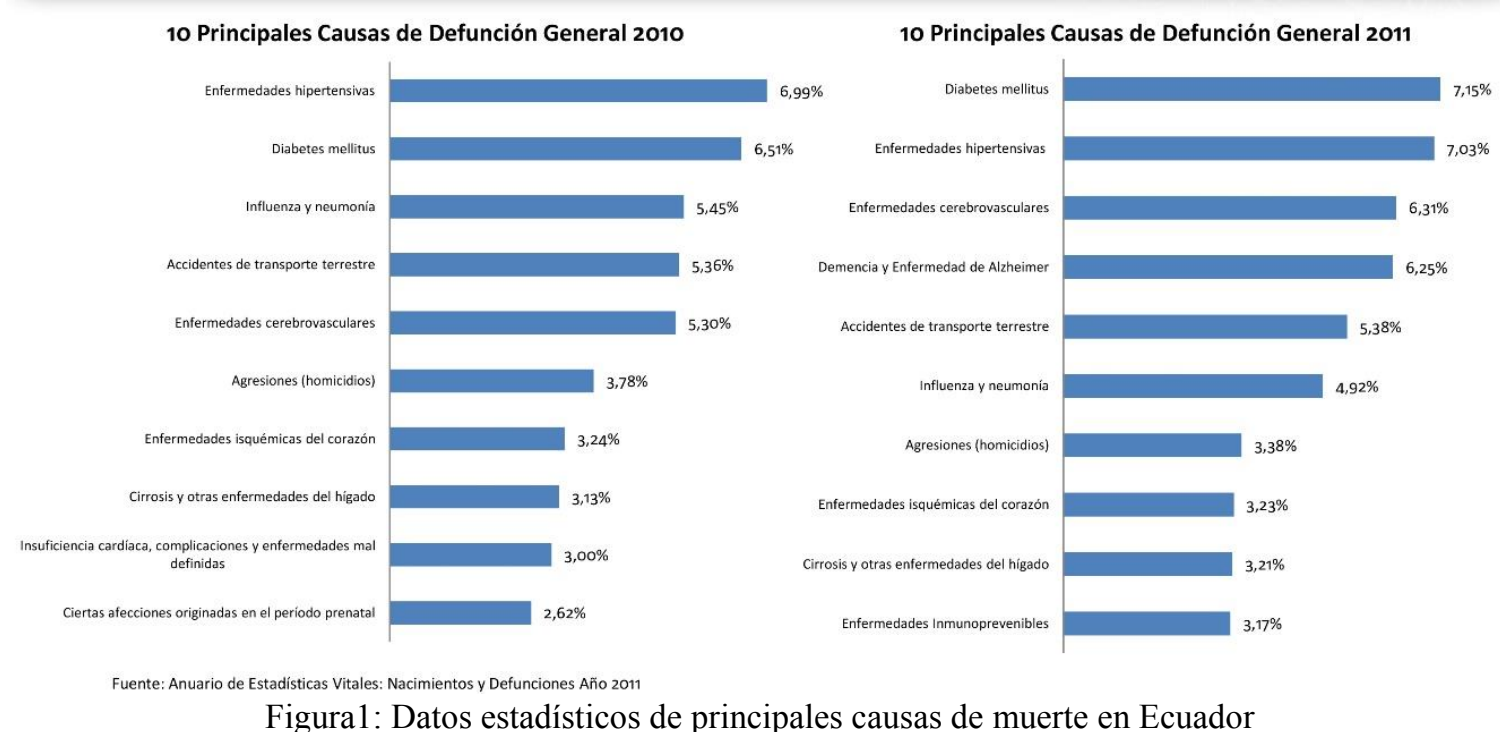

Fuente: INEC

En América latina Ecuador es el primer país que adoptó el sistema de semaforización en los productos alimenticios. La iniciativa surgió del éxito de la implementación de éste método en el Reino Unido. En el proceso educativo, el consumo responsable tiene como prioridad, proporcionar al público información necesaria para actuar de forma consciente con respecto a su alimentación.

Según el artículo 13 de la Constitución Ecuatoriana ${ }^{1}$

"Las personas y colectividades tienen derecho al acceso seguro y permanente a alimentos sanos, suficientes y nutritivos; preferentemente producidos a nivel local y en correspondencia con sus diversas identidades y tradiciones culturales. El Estado ecuatoriano promoverá la soberanía alimenticia." (p. 24).

\footnotetext{
${ }^{1}$ Constitución de la República del Ecuador. Véase en el siguiente enlace: http://www.asambleanacional.gov.ec/documentos/constitucion_de_bolsillo.pdf
} 
Por esta razón, el 18 de noviembre de 2013, de manera coordinada con dependencias del Ejecutivo a través del Ministerio Coordinador de Producción, Empleo y

Competitividad (MCPEC), de Industrias y Productividad (MIPRO), Coordinador de Desarrollo Social (MCDS), el INEN y el Agencia Nacional de Regulación, Control y Vigilancia Sanitaria (ARCSA), se extendió el Reglamento Sanitario de Etiquetado de Alimentos Procesados para el Consumo Humano ${ }^{2}$. (Cisneros, Trejo, \& Triviño, 2015).

Esta iniciativa busca ser otra alternativa para educar y concientizar al consumidor a corregir y/o modificar sus malos hábitos alimenticios para prevenir enfermedades, que al cambiar estas costumbres reducirían los altos niveles de mortandad en el país. Analizar el impacto de la implementación del semáforo nutricional en los hábitos alimenticios de los estudiantes de la Universidad Politécnica Salesiana, es el objetivo general de este proyecto investigativo.

Los objetivos específicos son:

- Establecer el nivel de conocimiento que tienen los estudiantes de la Universidad Politécnica Salesiana sobre el semáforo nutricional implementado en los productos alimentos procesados.

- Determinar el grado de afectación en las variables de sal, grasas y azúcares que inciden en la decisión de compra de un producto alimenticio procesado.

- Definir el alcance del semáforo nutricional en los estudiantes de la Universidad Politécnica Salesiana.

Si bien es cierto que Ecuador, es el primer país en América Latina en implementar esta nueva regularización, la importancia del presente trabajo investigativo, es demostrar el impacto que ha tenido en estudiantes universitarios esta metodología, además de conocer si está siendo utilizado de manera correcta a la hora de adquirir los productos alimenticios procesados.

\section{Etiquetado}

Este novedoso sistema de etiquetado indica los niveles: alto, medio o bajo contenido de grasas totales, grasas saturadas, azúcar y sal, para concientizar al consumidor sobre los correctos hábitos alimenticios que debe tener para prevenir enfermedades. El sistema que emplea este mecanismo de etiquetado que funciona a manera de semáforo de alerta, a través de los colores rojos, que es el alto contenido, el amarillo indicando el medio contenido, y el verde expresando el bajo contenido.

\section{Significado de los colores}

*Rojo: contiene un excesivo nivel de calorías, dañinos y perjudiciales para el cuerpo, es preferible evitar su consumo.

*Verde: este color hace referencia a aquellos alimentos que son bajos en calorías y se consumen diariamente

*Amarillo: color con el que se indica la mediana concentración de calorías,

\footnotetext{
${ }^{2}$ Reglamento sanitario de etiquetado de alimentos procesados para el consumo humano. Véase en: http://www.controlsanitario.gob.ec/wp-content/uploads/downloads/2014/08/Reglamento-sanitario-de-etiquetado-dealimentos-procesados-para-el-consumo-humano-junio-2014.pdf
} 


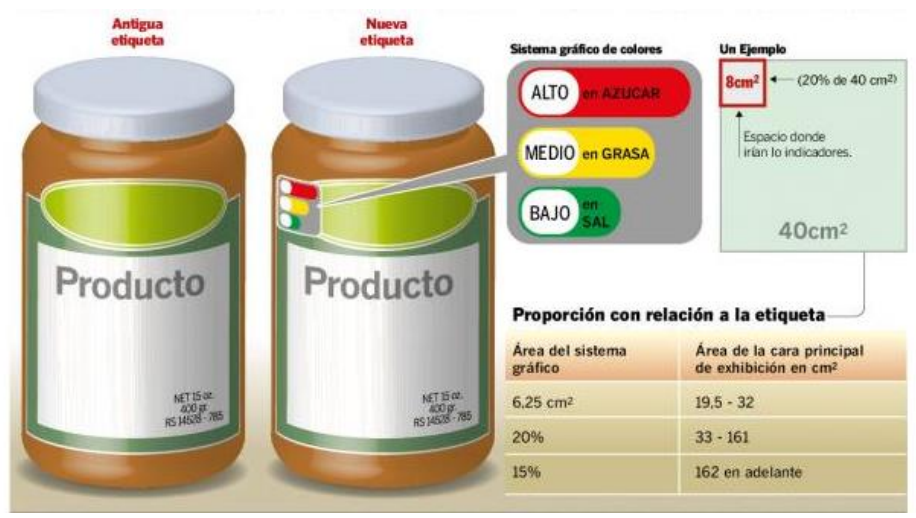

Figura 2: Colores de semaforización.

Fuente: Diario El Universo

\section{Regulación y control del etiquetado de los alimentos procesados.}

La Agencia Nacional de Regulación, Control y Vigilancia Sanitaria (ARCSA) es la institución encarga de inspeccionar la correcta implementación del sistema gráfico de etiquetado en todos los productos procesados, el mismo que debe ser colocado como adhesivo permanente, sellos indelebles o impresos en el rótulo que corresponde al producto. (Agencia Nacional de Regulación, Control y Vigilancia Sanitaria, 2014)

El ARCSA tiene como misión:

Contribuir a la protección de la salud de la población, a través de la gestión del riesgo de los productos de uso y consumo humano, así como de los establecimientos sujetos a vigilancia y control sanitario, facilitando a la vez el desarrollo del sector productivo nacional, entregando una atención ágil y expedita a los usuarios individuales e institucionales. (Agencia Nacional de Regulación, Control y Vigilancia Sanitaria, 2014)

Como agencia, el ARCSA revisa que las industrias alimenticias, apliquen la semaforización como fue normada en el reglamento sanitario de etiquetado de alimentos ${ }^{3}$ procesados para el consumo humano con la finalidad de mostrar una información oportuna, clara, precisa y autentica sobre el contenido y las características de los alimentos, para permitir la elección correcta para su adquisición. (Diario Hoy, 2013)

\section{Impacto social del semáforo nutricional}

El impacto generado por la implementación del etiquetado del semáforo nutricional fue tanto en los consumidores como en las empresas del sector de la producción alimenticia. El consumidor por un lado sabiendo que hay una manera de conocer que productos afectan la salud en el momento de adquirirlos, guiándose por los colores rojo, amarillo y verde que muestran el grado que contienen los alimentos en escala de bajo, medio y alto en grasas, sales y azúcares para así saber que alimentos deberían evitar o eliminar de la dieta diaria, cuales pueden consumir diariamente, y cuáles deben ser consumidos de manera moderada.

En el sector de la producción alimenticia su impacto fue muchos más fuerte debido a que

\footnotetext{
${ }^{3}$ Ejemplo de etiquetas. Véase en: http://elpoderdelconsumidor.org/wpcontent/uploads/2014/06/EcuadorEtiquetadoEjemplos.pdf
} 
todas las empresas de esta sección se vieron en la obligación de implementar esta nueva normativa.

Uno de los segmentos más afectados fue el de los lácteos y sus derivados, mientras que para otros el impacto generado no causó bajas representativas en sus ventas, si debieron invertir una fuerte cantidad de dinero en realizar los cambios en la etiqueta para cumplir con la normativa impuesta, otros debieron ofertar productos más saludables para no verse afectados. (Comercio, 2014)

\section{Metodología}

En este proceso investigativo se procederá a utilizar una metodología cuantitativa y cualitativa, convirtiéndolo así, en un estudio pluriparadigmático. Además, se desarrollará dentro de los parámetros explicativos, permitiendo convertirse en una guía para el estudio y descripciones de terminologías referente al presente trabajo.

Para (Pérez \& Porras, 2015), dentro del proceso pluriparadigmático existe una posibilidad de coexistencia de una multiplicidad de paradigmas, que a su vez potencian el surgimiento de una trama de interacciones entre los estatutos epistemológicos y las metodologías emergentes (pág. 105). Por esta razón, no solo es importante investigar con esta perspectiva, ya que permitirá unir los dos métodos para obtener resultados más amplios.

Dentro del enfoque cuantitativo, como método empírico, se hará uso la encuesta.

Para Bueno (2003) "Es uno de los métodos que permite obtener información sobre fenómenos y procesos, que no pueden ser adquirida a partir de la observación directa ni de los distintos documentos existentes" (p.70). Los datos estadísticos juegan un papel muy importante dentro del estudio ya que será un elemento complementario durante la recolección de datos.

Por otro lado, el enfoque cualitativo para Corbetta (2003) citado en (Hernández, Fernández, \& Baptista, 2010) es "el enfoque cualitativo evalúa el desarrollo natural de los sucesos, es decir, no hay manipulación ni estimulación con respecto a la realidad" (p. 9); lo que hace referencia a una valoración sobre la implementación del semáforo nutricional en los productos procesados dentro del Ecuador. Para (Galindo, 1998), esta técnica la podemos definir como “... una conversación verbal entre dos o más seres humanos (entrevistador y entrevistado), cuya finalidad es lo que en verdad le otorga tal carácter" (p. 281).

Cabe mencionar que las entrevistas se realizarán a personas expertas en el tema (doctores) y consumidores de productos alimenticios procesados, mientras que las encuentras se realizará a la muestra de estudiantes seleccionada. En la presente investigación el proceso de muestreo se calculó con un 95\% de confianza y un margen de error del 5\% con un tamaño poblacional de 7.722 estudiantes con edades que varían entre los 17 y 33 años, dentro de ella se encontró diversos grupos etarios que formaron parte de esta selección. Para ello se recurrió a la siguiente fórmula:

$$
n=\frac{z^{2}\left(p^{*} q\right)}{e^{2}+\frac{\left(z^{2}\left(p^{*} q\right)\right)}{N}}
$$

Donde: 
$\mathrm{n}=$ Tamaño de la muestra

$\mathrm{z}=$ valor asociado al nivel de confianza $(1,96)$

$\mathrm{p}=$ Proporción de la población con las características deseadas (éxito) (0.5)

$\mathrm{q}=$ Proporción de la población sin la característica deseada (fracaso) $(0.5)$

$\mathrm{e}=$ Nivel de error dispuesto a cometer $(5 \%)$

$\mathrm{N}=$ Tamaño de la población (7722 estudiantes registrados)

\section{Resultados}

Dentro de este ítem se expondrá el análisis de las técnicas empleadas para lograr dar respuestas a los objetivos planteados en la presente investigación. En primera instancia presentarán los datos de las encuestas y en segunda lugar las entrevistas. El diagnóstico realizado fue el eje conductor del proceso desarrollado.

La encuesta, fue utilizada con el objetivo de conocer la información que manejan los estudiantes de la Universidad Politécnica Salesiana sede Guayaquil con respecto al semáforo nutricional. La aplicación de esta técnica mostró los siguientes resultados: la primera pregunta se planteó con la finalidad de estar al tanto del conocimiento que tienen los estudiantes de la Universidad Politécnica Salesiana acerca del semáforo nutricional.

Los resultados fueron alentadores al notar que el 58\% de los encuestados tenía conocimiento de esta normativa para controlar la ingesta de productos dañinos para la salud. (Ver figura 4)

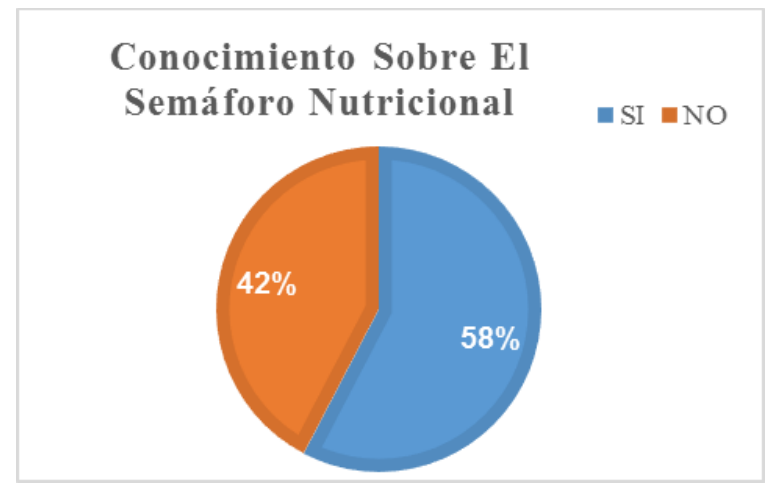

Figura 4: Conocimiento sobre el Semáforo Nutricional

Fuente: Elaboración propia

Con la segunda pregunta lo que se pretendió fue establecer cuántos alumnos sabían la utilidad del Semáforo nutricional dando como resultado que el 57\% (ver figura 5) sabía el beneficio de esta nueva metodología para mejorar o hacer cambios en los hábitos alimenticios, mientras que el $43 \%$ no. 


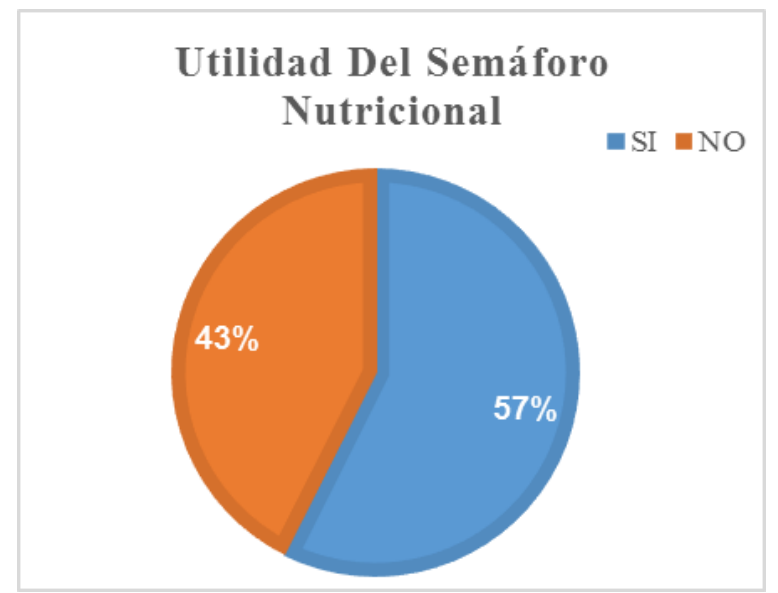

Figura 5: Utilidad del Semáforo Nutricional

Fuente: Elaboración propia

Con el objetivo de conocer si los consumidores, estudiantes de la Universidad Politécnica Salesiana sede Guayaquil, revisan los niveles descritos en el semáforo antes de su compra, se planteó la tercera interrogante. Ante ella la respuesta que se obtuvo fue que el 51\% no inspeccionan los productos ya sea por descuido, porque eligen lo que les gusta, etc., mientras que $49 \%$ si lo hace. (ver figura 6 )

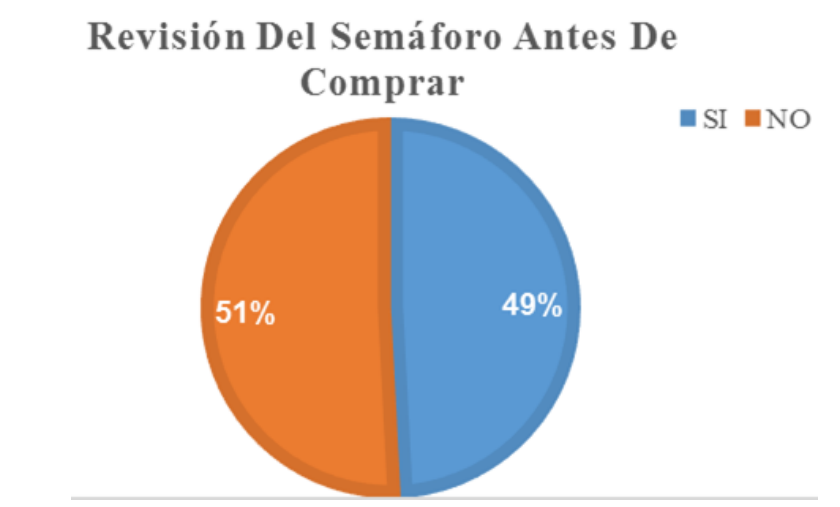

Figura 6 Revisión del semáforo antes de comprar un producto

Fuente: Elaboración propia

Por otro lado, con la intención de saber cuál era el producto sanforizado que más consumían los encuestados, se realizó la cuarta pregunta. En este planteamiento se conoció que el $60 \%$ no tiene un producto definido, mientras que el $40 \%$ restante coincidía en que los que más se fijaban eran los snacks, los lácteos y las bebidas gaseosas. (Ver figura 7) 


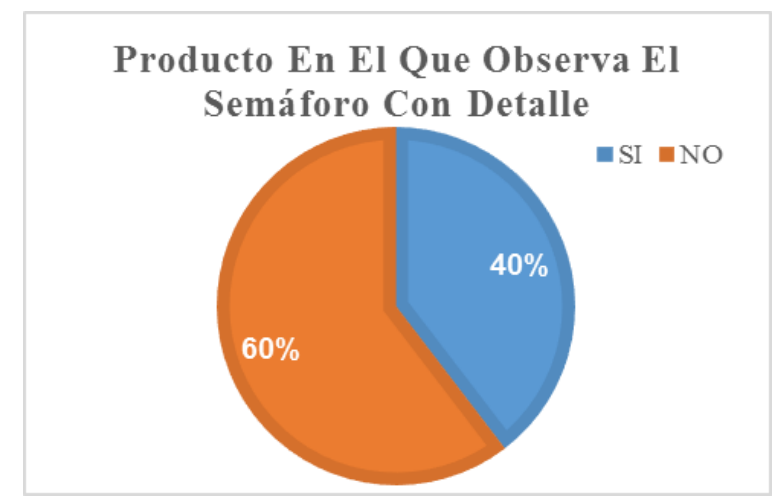

Figura 7: Producto en el que observa el semáforo con detalle

Fuente: Elaboración propia

Con la quinta pregunta logramos obtener los resultados de uno de los objetivos específicos descritos en el presente proyecto. Determinar el grado de afectación en los variables de sal, grasas y azucares que inciden en la decisión de compra de un producto alimenticio procesado, logrando como respuesta que para el 40\% el nivel de azúcar del producto es la variable más importante que incide en su decisión de compra, seguido con el 34\% de grasas y el $26 \%$ de sales siendo este factor el que menos influencia en la decisión de compra. (Ver figura 8).

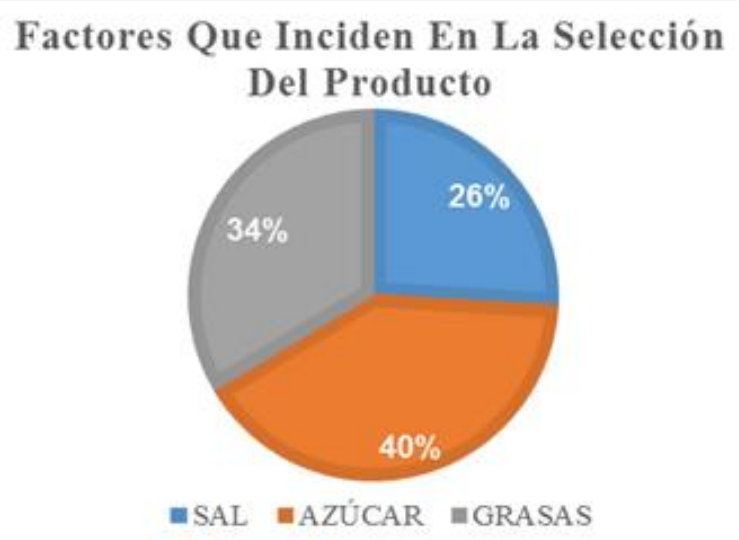

Figura 8: Factores que inciden en la selección del producto

Fuente: Elaboración propia

Con la sexta y última pregunta se logró saber si la implementación del semáforo nutricional provocó un impacto en los hábitos alimenticios de los estudiantes de la Universidad Politécnica Salesiana sede Guayaquil, teniendo como respuesta que el 40\% respondió que si había generado mucho impacto de tal manera que habían realizado cambios en su forma de alimentarse, el 39\% indico que los cambios se han realizado debido a que el semáforo era poco tomado en cuenta a la hora de comprar, y finalmente el $21 \%$ indicó que no había generado ningún tipo de cambio en sus hábitos alimenticios, porque ya están habituados a comer de esa manera. (Ver figura 9) 


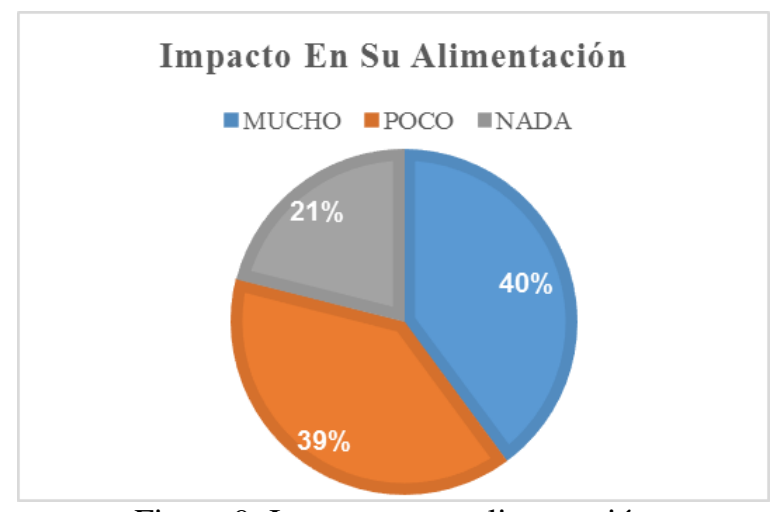

Figura 9: Impacto en su alimentación

Fuente: Elaboración propia

Dentro de la metodología cualitativa se utilizó la entrevista, las mismas que se aplicaron a profesionales de la salud y personas dentro de los centros comerciales con el objetivo de conocer si aplican o no la semaforización de los productos a la hora de adquirirlos, obteniendo los siguientes resultados: Para la Dra. Agheda García, médico del Hospital Efrén Jurado López, la implementación del semáforo se realizó sin una previa campaña educacional a los consumidores, es por ese motivo que la aplicación del semáforo no ha generado la respuesta deseada por parte de los entes reguladores. Dentro de este marco el Sr. Carlos Castillo coincidió con la especialista en la falta de formación en la sociedad con respecto a esta nueva normativa.

Por otro, los entrevistados manifestaron que es necesario realizar unos ajustes al semáforo nutricional debido a que es insuficiente sólo mostrar los niveles altos, medios, bajos en grasas, sales y azúcares, cuando el producto puede tener otros elementos que sean perjudiciales para la salud y no se muestran en la semaforización.

Con respecto a la semaforización, se manifestó que, si bien es cierto, esta herramienta permite guiar al comprador bajo las características ya mencionadas, no existe una tabla que indique la cantidad exacta de elementos, por ejemplo: grasas saturadas o calorías que se deben controlar dentro de la alimentación diaria de las personas. Permitiendo así, el incremento de enfermedades no transmisibles como la diabetes, la obesidad, entre otras.

Con relación al impacto que ha tenido el semáforo nutricional, los entrevistados coinciden en que no ha causado la reacción esperada del público, ya que los consumidores eligen sus productos por gusto más no por la información alimenticia que pueden tener. Sin embargo, existe un bajo porcentaje de personas que si han sido influencia por esta metodología dentro de sus hábitos alimenticios.

\section{Discusiones}

En la presente investigación se pudo comprobar el alto impacto que ha ocasionado la implementación del semáforo nutricional en los consumidores de la muestra seleccionada que estuvo integrada por los estudiantes de la Universidad Politécnica Salesiana sede Guayaquil. Durante el desarrollo de la investigación, se constató una gran diferencia entre el proceso de semaforización existente en países desarrollados como el Reino Unido, España entre otros. En estas naciones, este proceso es mucho más específico que el empleado en Ecuador, en ellos 
existe una mayor información de la cantidad exacta de los elementos que deben de ser controlado para evita enfermedades.

Sin embargo, actualmente según Pedro Maldonado, Coordinado de la Revista Líderes, el Ministerio de Industria y Productividad (MIPRO), no eliminará el semáforo nutricional, pese a la poca utilidad que se le da en este momento, sino que se modificará ${ }^{4}$ con la cantidad recomendada de grasas, azúcares y sales que deben ser ingeridos con respecto a las necesidades diarias de cada persona.

Por otro lado, según el Gobierno Nacional del Ecuador, si se ha realizado una correcta difusión ${ }^{5}$ de este nuevo proceso que inició en el 2013 tanto medios audiovisuales como en medios impresos y radiales, pero de acuerdo a las personas entrevistadas estas campañas debieron realizarse antes, durante y después con la misma intensidad para dar tiempo a que todos los consumidores se eduquen a esta nueva normativa en pro de la salud.

De acuerdo a la implementación del semáforo nutricional como pionero en Latinoamérica, Ecuador posiciona su marca país ${ }^{6}$, en la lucha por crear conciencia en los consumidores con respecto a la importancia de modificar los hábitos alimenticios de toda una sociedad para disminuir las altas tasas de mortandad causadas por las enfermedades no transmisibles que podrían ser evitadas, si como consumidores controláramos más la ingesta de productos dañinos para la salud.

\section{Conclusiones}

Los resultados obtenidos muestran que los estudiantes de la Universidad Politécnica Salesiana presentan un nivel alto de información acerca del semáforo nutricional los mismos que realizan una mejor elección de los productos que compran gracias a la observación del mismo. Hay quienes coinciden en que se debió y se debe continuar con campañas educacionales que indiquen cómo se usan los colores, que implica que sea alto en grasa, sales y azúcares para que el etiquetado de semáforo nutricional, pueda generar el impacto que buscaron el Gobierno Nacional y demás entes reguladores para que el consumidor modifique sus hábitos alimenticios y así poder prevenir y reducir las altas tasas de mortandad en el país por las enfermedades no transmisibles.

Ecuador como marca país podrá posicionarse aún más, cuando se demuestre estadísticamente como la aplicación de la semaforización nutricional, ayudó a disminuir las tasas de mortandad por las enfermedades no transmisibles, gracias a la modificación de los hábitos alimenticios de la sociedad ecuatoriana.

\footnotetext{
${ }^{4}$ Revista Líderes:

https://www.youtube.com/watch?v=avSEc5ndUkY\&ebc=ANyPxKqujasI6oORsFbwtYO2G9fQaoBpNvBG7BZyO8 XzhG2bMLdmUf-9aV9XxED5xHZOcBwNx7jCn0cpE23vUODxDZvh2gRLjA

${ }^{5}$ Autoridades informando a la sociedad sobre el semáforo nutricional: https://www.youtube.com/watch?v=vj_QVVZIT7g

Campaña realizada por el gobierno: $\mathrm{https} / / \mathrm{www}$.youtube.com/watch?v=LekdMEaETZw

Declaraciones del Presidente del Ecuador con respecto al semáforo nutricional: https://www.youtube.com/watch?v=gJvAzAF7zo8\&ebc=ANyPxKpZFqysdf1U138T7fU5oJYjBv9ukTVXeuUB_8V bJjMAlzCCM3ECS7DfiyRHGFizNcchQqbHVq8C2I1kXkYhfsSpemc8yA

${ }^{6}$ Marca país es una estrategia para capitalizar la reputación de un país en mercados internacionales. (Echeverri Cañas, s.f.) https://paismarca.com/que-es-marca-pais-2/
} 
Este proyecto investigativo demostró que la implementación del semáforo nutricional, si generó modificaciones en sus hábitos alimenticios, la gran mayoría en el consumo de los snacks y las bebidas gaseosas, debido a que este método les ayuda a saber si lo que van a consumir es alto en grasas y azúcares que fueron los indicadores determinantes en el momento de seleccionar un producto. A pesar de que Ecuador fue en país pionero en América latina en implementar esta herramienta, no cabe duda que aún falta más trabajo para concientizar a los consumidores del peligro de ciertos alimentos, además deben incrementar la información que reciben los usuarios en el etiquetado semaforizado de los productos procesados.

\section{Bibliografía}

Aguirre Choix, R., \& Armenta Velazquez, C. E. (2012). La importancia del control interno en las pequeñas empresas en México. El Buzón de Pacioli, Año XII Número 76 Enero-Marzo , 1-17.

Albanese, D., \& Boland, L. (2004). PyMes: algunas herramientas para diagnosticar su problemática. Escritos Contables. n.45 Bahía Blanca , 49-62.

Amaro Zárate, B. I. (2003). Valor Económico Agregado como estrategia financiera en las PYMES del sector automotriz. Puebla: Universidad de las Américas Puebla.

Andersen , A. (1999). Diccionario de Economía y Negocios. España: Espasa.

Araque J., W. (2012). Las PyME y su situación actual. Quito.: Observatorio PYME. Universidad Andina Simón Bolívar.

Bueno Campos, E. (2007). Organizacion de empresas: estructura, procesos y modelos (2a ED.) . Madrid: Piramide.

Duff, V., \& Media, D. (2016). Toma de decisiones gerenciales. La Voz de Houston and the Houston Chronicle, págs. http://pyme.lavoztx.com/toma-de-decisiones-gerenciales-4091.html

Fundéu-BBVA. (2011). Fundéu-BBVA. Recuperado el 22 de Junio de 2016, de http://www.fundeu.es/recomendacion/pyme-no-pyme-ni-pyme-1049/

García Caraballo, J., \& Macías Mesa, J. A. (2010). El Proceso de Inteligencia Empresarial en las Empresas del Grupo de Diseño e Ingeniería de la. Revista de Arquitectura e Ingeniería, vol. 4, núm. 2, Versión On-line, disponible en: http://www.redalyc.org:9081/home.oa?cid=444279

García Del Junco, J., \& Casanueva Rocha, C. (2001). Prácticas de la gestion empresarial. Madrid: Mc Graw Hill.

Garza Ríos, R., González Sánchez, C., Pérez Vergara, I., Martínez Delgado, E., \& Sanler Cruz, M. (2012). Concepción de un procedimiento utilizando herramientas cuantitativas para mejorar el desempeño empresarial. Ingeniería Industrial. Vol.33 no.3, versión On-line ISSN 1815-5936.

González Jaramillo, R. (2013). Las MIPYMES ecuatorianas dinamizan la economía nacional. En H. J. King, Estudios industriales de la micro, pequeña y mediana empresa (págs. 16 - 18). Quito: FLACSO. 
Herranz, A. (12 de Septiembre de 2013). Cómo mejorar la toma de decisiones en la pyme. Recuperado el 22 de Junio de 2016, de http://www.ticbeat.com/pymes/como-mejorar-la-toma-dedecisiones-en-la-pyme/

INEC. (2010). Censo Nacional Económico . Quito: Instituto Nacional de Estadísticas y Censos .

Instituto Nacional de Estadísticas y Censos. (2014). Directorio de Empresas y Establecimientos (DIEE). Quito: INEC.

Lemes Batista, A., \& Machado Hernández, T. (2007). Las pymes y su espacio en la economía Latinoamericana. Segundo Encuentro Internacional sobre las Medianas, Pequeñas y MicroEmpresas del Siglo XXI. On-line: Eumed.net.

Méndez Morales , J. S. (1996). Economía y la Empresa. México: McGraw-Hill.

Miller Franco, L., Newman, J., Murphy, G., \& Mariani, E. (1997). La resolución de problemas y el mejoramiento de procesos como medios para lograr calidad. Wisconsin : Center for Human Services.

Navarrete Báez, F. E. (2013). Las Micro, Pequeñas y Medianas Empresas de a Zona Metropolitana de Guadalajara: una perspectiva hacia la gestión de su proceso de toma de decisiones. Revista Electrónica Nova Scientia.Vol 5, num 10, 210 - 236.

Ordóñez Islas, P. A. (2012). Metodología para el diagnóstico organizacional de PYMES (Tesis). México, D. F.: Universidad Nacional Autónoma De México.

Porter, M. (2003). Ser competitivo: nuevas aportaciones y conclusiones. Barcelona: DEUSTO S.A. .

Rodríguez Valencia, J. ( 2002). Administración de pequeñas y medianas empresas, 5ta Edición. México: Thomson.

Romero, R. (1999). Marketing. Palmir E.I.R.L.

Ross, A. G., Zeballos, J. L., \& Infante, A. (2000). La calidad y la reforma del sector de la salud en América Latina y el Caribe. Revista Panamericana de Salud Pública.vol.8 n.1-2, On-line version ISSN 1680-5348.

Secretaría Nacional de Planificación y Desarrollo. (2012). Plan Nacional de Desarrollo, Plan Nacional para el Buen Vivir 2009-2013: Construyendo un Estado Plurinacional e Intercultural. . Quito: SENPLADES.

Universidad San Martín de Porres. (2012). Organización y gestión de PYMES. Perú: Universidad San Martín de Porres. Facultad de Ciencias Administrativas y Recursos Humanos.

Velasco, C. (9 de Febrero de 2010). La toma de decisiones del emprendedor a cargo de una Pyme. Coyuntura Económica, págs. http://coyunturaeconomica.com/emprendimiento/toma-dedecisiones-pyme. 